\title{
New Canadian Initiatives in Dementia
} Research

Today, as a result of the relative paucity of research funds available from universities, hospitals, charitable organizations, governments and the Medical Research Council, a link to the private sector is a financial lifeline for many departments of neurology, psychiatry and geriatrics. Fortunately, the pharmaceutical industry has expanded its Phase II and Phase III trial activity in Canada in recent years, and increasingly appears to be turning to academic centres for conceptual advice as well as for that practical support which, uniquely, they can provide. Within the field of dementia, the clinical trials for which academic centres contract demand precise, reliable, repetitive assessment of subjects by clinicians, psychologists, nurse-coordinators and caregivers. But every physician knows that the number of patients that actually enrolled in clinical trials represents less than a fifth of those initially thought to be eligible. And many physicians who are engaged in this necessary work are too often perturbed by the anonymous, inflexible or arbitrary protocol imposed by the pharmaceutical sponsor, clinical trial physicians often lacking the opportunity to participate meaningfully in study design and thus limited to providing their expertise in assessment of a drug which they did not produce, using methods of which they do not necessarily approve.

Either of two unwanted situations might evolve in these circumstances. The first is the performance of contract research projects solely in the largest institutions; the second is the agreement of a smaller centre recruited to provide a larger number of subjects for a trial than it can ultimately enter, which is annoying to the sponsor and potentially a financial embarrassment for the centre itself. The situation requires improvement; in certain research areas, clearly this is being achieved.

The Canadian National Cancer Centre and the HIV Trials Networks should be of interest to neurologists. In each case, relying (initially at least) upon government support, these groups have been successful in identifying, recruiting and sustaining cooperative activity within Canadian centers, such that their staffs can feel a permanent part of a National research strategy, can work with their colleagues in the study of diseases according to protocols generated within their own group, can add studies of academic importance to the commercial protocols, and can interact in order to provide national standards for all aspects of the disorder question. In a similar American model, the research to be performed is actually contracted to the clinical trials organization, which thus becomes responsible for the proper performance of the trial. It is a question of some ethical validity as to whether the institution synthesizing a putative therapeutic substance should also be that appointed to determine its effects. But at present, for many clinical departments, thera- peutic trials act as an economic powerhouse, while the add-on studies represent the individual, academic components of the work which, as a result, goes faster and allows the study physicians a sense of personal contribution.

Three years ago, the Consortium of Canadian Centres for Clinical Cognitive Research $\left(\mathrm{C}_{5} \mathrm{R}\right)$ was founded by a group of Canadian neurologists, psychologists, psychiatrists and gerontologists. Fortuitously at that time the Canadian Study of Health and Aging had identified its potential members, who represent such disciplines across the country and among whom there was then surfacing an element of disenchantment with the purely passive rôle of 'principal investigators' in the therapeutic trials of cognitively-active agents. The visible result to date has been the publication of two notable papers in this Journal; ${ }^{1,2}$ the completion of studies of great academic interest added-on to therapeutic trial protocols; two annual satellite symposia held in association with the Canadian Congress of Neurological Sciences; and plans for formal linkage of study sites for future basic, clinical and therapeutic research projects.

Canadian workers in the fields of stroke and epilepsy have noted this initiative, and may emulate it; one day, others engaged in such fields as involuntary movement disorders, peripheral neuropathy, myopathy, neurogenetics and more besides might decide to take the same route, because the example of the $C_{5} R$, even in its early stages, as an academic thinktank and as a research network is already impressive.

One must hope that the invitation of Rockwood and his colleagues ${ }^{1}$ to Canadian neurologists and others to validate a set of diagnostic criteria for the vascular dementias will be accepted; if physicians working in different disciplines in many Canadian regions will only provide the information sought in the simple if extended checklist presented, then the pressing questions as to the nature, the semeiology and the provenance of the vascular dementias might be answered by a country, when any single centre can but remain silent; there is truth in numbers, and the greater numbers, the more valid the truth. This may be considered the first public initiative of the $\mathrm{C}_{5} \mathrm{R}$, although its formal participation in National Seminars on aspects of the dementias has already made it visible.

In this issue of the Journal, ${ }^{2}$ Mohr, Feldman and Gauthier break new ground in proposing guidelines for the development of anti-dementia therapies, advocating certain diagnostic or assessment schedules in cognitive disease protocols in preference to others, proposing further cooperative studies in certain areas such as the non-Alzheimer dementias, emphasizing the importance of behavioral and functional as well as cognitive assessments, making reasonable recommendations on the place 
of imaging studies in the context, and separating the domains of cognition, behaviour and function as areas worthy of assessment independently in the process of determining the efficacy of therapeutic agents. They also call into question some of the dogmas of today, such as the Clinical Interview Based Impression of Change, a subjective and quite unvalidated scale, universally employed only because of enforcement by American regulatory personnel. If this trend prevails, then the responsibility for the design and perhaps the execution of clinical trials might revert to those expert and disinterested clinicians active in the field, leading a consortium. Its warm reception of the aims of the $C_{5} R$ and the other academic or trials networks says much for the ethicism as well as the farsightedness of the Canadian pharmaceutical industry.

The authoritative statements from the Consortium published in the Journal ${ }^{1,2}$ are important because they demonstrate that interest in the dementias in Canada is sophisticated, innovative and organized, and because they provide a multidisciplinary, trans-national consensus viewpoint. The initial arms-length support by the pharmaceutical industry has enabled the consortium to frame research guidelines for diagnosis and investigation of the dementing diseases. The prevalence figures derived from the Canadian Study of Health and Aging ${ }^{3}$ allow no doubt that the subject is immediate; such initiatives make it also an increasingly interesting field of study, into which this country would be well advised to channel more people, more energy and more resources; because the demography of disease in the elderly Canadian population of tomorrow demands it.

William Pryse-Phillips

St. John's, Newfoundland

\section{REFERENCES}

1. Rockwood K, Parhad I, Hachinski V, et al. Diagnosis of Vascular Dementia: Consortium of Canadian Centres for Clinical Cognitive Research Consensus Statement. Can J Neurol Sci 1994.

2. Mohr E, Feldman H, Gauthier S. Canadian Guidelines for the Development of Antidementia Therapies: a Conceptual Summary. Can J Neurol Sci 1995; 22: 62-71.

3. Canadian Study of Health and Aging. Methodology and Prevalence Data. Can Med Assoc J 1994; 150: 899-913. 